\title{
TITANIUM DIOXIDE SUSPENSION PHOTOCATALYSIS IN A TUBULAR PHOTOREACTOR AND TITANIUM DIOXIDE REUSE ON THE REMOVAL OF PHENOL
}

\author{
By : Dhanus Suryaman \\ Agency for the Assessment and Application of Technology
}

\begin{abstract}
We studied flow rate, electrolytes effects, titanium dioxide separation and reuse and sunlight application on photocatalysis in a tubular photoreactor integrated with the titanium dioxide separation/reuse. The circulative flow treatment was preferred over the single pass treatment. Phenol and TOC removals in tap and deionized waters were a maximum at the circulative flow rate of $600 \mathrm{ml} \mathrm{min}^{-1}$ and the transmittance of $0.26 \%$. At low flow rate, titanium dioxide particles were sedimented in tap water, but those were perfectly suspended in deionized water. The titanium dioxide slurry was sedimented spontaneously by standing and was repeatedly reused without decreasing the degradation efficiency.
\end{abstract}

Keywords : Photocatalytic, Optimum flow, Electrolytes, Separation, Solra

\section{INTRODUCTION}

Titanium dioxide $\left(\mathrm{TiO}_{2}\right)$ is one of the basic materials in everyday life. It has been widely used as white pigment in paints, cosmetics and foodstuffs. $\mathrm{TiO}_{2}$ exists in three crystalline modifications: rutile, anatase, and brookite. Generally, $\mathrm{TiO}_{2}$ is a semiconducting material which can be chemically activated by light. Under the influence of light, the material tends to decompose organic materials. Although $\mathrm{TiO}_{2}$ absorbs only approximately $5 \%$ of the solar light reaching the surface of the earth, it is the best investigated semiconductor in the field of chemical conversion and storage of solar energy. In recent years semiconductor photocatalysis using $\mathrm{TiO}_{2}$ has been applied to important problems of environmental interest like detoxification of water and air.

The $\mathrm{TiO}_{2}$ is the most often used photocatalyst due to its considerable photocatalytic activity, high stability and low cost. The supported $\mathrm{TiO}_{2}$ photocatalytic treatment is easily operated, however, it requires a complicated preparation of the $\mathrm{TiO}_{2}$ immobilization and has $\mathrm{TiO}_{2}$ detachment problems during long-term applications (1). The $\mathrm{TiO}_{2}$ suspension method excels over the immobilized or supported $\mathrm{TiO}_{2}$ method with respect to the efficiency of the photocatalytic degradation (2), however, it requires separation after the photocatalytic treatment.

On the other hand, the real wastewater ordinarily contains more abundant electrolytes than the original water, which lower the degradation efficiency due to the coagulation of $\mathrm{TiO}_{2}$ (3,4). However, sufficient caution has not been paid to the degradation experiments of water pollutants even in those directed toward pilot plants.

We have reported that the electrolytes in river and tap waters produce a slightly decreased efficiency, while they enabled easy separation of the fine $\mathrm{TiO}_{2}$ particles using a solids-retaining centrifuge, which was combined with a batch photoreactor (4). However, it was necessary to remove the accumulated $\mathrm{TiO}_{2}$ from the rotor (2 I) by interrupting the operation for every 1315 Itreatment of the model wastewater. Reuse of the recovered $\mathrm{TiO}_{2}$ has been reported after neutralization-sedimentation ${ }^{(3)}$ and microfil tration (5) by the repeated treatment.

These processes do not allow a continuous wastewater treatment and a fast, cheap and easy separation process. A high circulative flow rate has been used to pump the $\mathrm{TiO}_{2}$ suspension into a tubular photoreactor $(3,6,7)$, however, they did not investigate the effects of electrolytes in the water and flow rates on the photocatalytic efficiency. The photocatalytic degradation of phenol, a commonly found pollutant in industrial waste effluent, using $\mathrm{TiO}_{2}$ has been studied (8-10).

Therefore, we studied the flow photocatalysis in the tubular photoreactor integrated with the $\mathrm{TiO}_{2}$ separation and reuse for degradation of phenol in the electrolytecontaining model wastewater (tap water). The effects of flow rate and electrolytes, $\mathrm{TiO}_{2}$ separation and reuse and application of sunlight were investigated. The reuse of the $\mathrm{TiO}_{2}$ particles for repeated photocatalytic treatments promotes the application of the $\mathrm{TiO}_{2}$ suspension than those of the immobilized. A symptom of the $\mathrm{TiO}_{2}$ suspension in tap water in the tubular photoreactor was observed in this study. 


\section{EXPERIMENTAL}

All chemicals were of reagent-grade quality and were used as received. The phenol solutions were prepared with tap water from Toyama city. The electrolytes $\left(\mathrm{mg} \mathrm{l}^{-1}\right)$ in the tap water were as follows: $\left[\mathrm{Na}^{+}\right]=1.9, \mathrm{~K}^{+}=0.5, \mathrm{Ca}^{2+}$ $=7.8, \mathrm{Mg}^{2+}=1.0, \mathrm{Cl}^{-}=3.3, \mathrm{SO}_{4}{ }^{2-}=9.0, \mathrm{HCO}_{3}{ }^{-}=$ 18.9. Deionized water was used when examining the effect of the electrolytes on the photocatalytic degradation of phenol and coagulation.

Figure 1 shows a schematic of the photocatalytic treatment- $\mathrm{TiO}_{2}$ separation system. The photocatalytic treatments were carried out in the tubular photoreactor by varying the flow rate or irradiation time. Pyrex tubes (24 pieces, 1 piece: $75 \mathrm{~cm}$ length and $0.6 \mathrm{~cm}$ i.d., total volume: $510 \mathrm{ml}$ ), of which each end was attached to tygon tubes, were installed onto a stainless steel plate $(200 \mathrm{~cm} \quad 100 \mathrm{~cm})$ and furthermore, the head and tail parts of the combined pyrex tubes were combined with a tygon tube covered black hose (volume: $285 \mathrm{ml}$ ). Irradiation was performed with sunlight or black light lamps $\left(2020 \mathrm{~W}, \lambda_{\max }: 352\right.$ $\mathrm{nm})$.

Black light irradiation was performed inside a surrounding wall of which the inside is covered with reflecting stainless steel sheets. A top panel covered stainless steel sheets, under which the black lights were attached, was inserted inside the surrounding wall and held 20 $\mathrm{cm}$ above the tube with plugs set inside the wall. Sunlight irradiation was performed by removing the surrounding wall and the lamps attaching top panel. The mixing tank $(31 \mathrm{~cm}$ height and $21 \mathrm{~cm}$ i.d.) was equipped with a mechanical stirring and covered with black flannel. The light intensities of the black light and sunlight were measured by an illuminometer (ORC UV-MO2) at the wavelength of $320-390 \mathrm{~nm}$ and estimated to be $1.7 \mathrm{~mW} \mathrm{~cm}^{-2}$ and $2.9-3.3 \mathrm{~mW} \mathrm{~cm}^{-2}$, respectively.

The $\mathrm{TiO}_{2}$ particles of Degussa P-25 (Nippon Aerosil Co., 15-40 nm particle size) were used as a photocatalyst. The $\mathrm{TiO}_{2}$ particles (5.0 g) were added to the phenol solution (10 I) in the mixing tank and mechanically stirred. The flow of the $\mathrm{TiO}_{2}$ suspension and slurry was adjusted by a rolled pump. The photocatalytically treated effluents were stored in a separation tank (10.7 I) and overflowed. In all the experiments, the $\mathrm{pH}$ of the phenol solutions was not adjusted during the course of the experiment.

About $2 \mathrm{ml}$ of the effluents that flowed out from the photoreactor were withdrawn at timed intervals and immediately filtered using a syringe equipped with a disposal filter having a pore size of $0.2 \mathrm{~m}$. The concentration of TOC in the water was measured by a Shimadzu TOC500 analyzer. The concentration of phenol was measured by an HPLC system equipped with a PU-980 pump, a 970 UV-Vis detector (Jasco), and a Mightysil RP-18 column (Kanto Chemicals). The measurement was made using the mobile phase of $\mathrm{CH}_{3} \mathrm{CN}: \mathrm{NaH}_{2} \mathrm{PO}_{4}(20 \mathrm{mM})$ $=50: 50$ at the wavelength of $215 \mathrm{~nm}$. The transparency of the treated water was measured at $400 \mathrm{~nm}$ by a UV-1600 Shimadzu spectrophotometer.

\section{RESULTS AND DISCUSSION}

Figure 2 shows the effects of the flow rate or irradiation time on the degradation of phenol and the transmittance of the effluent flowing from the photoreactor. When deionized water was used, the decrease in the phenol concentration can be approximated by an exponential curve. As comparison, a previous study (8) reported a low degradation of phenol in distilled water in a batch cylinder photoreactor under no stirring. When tap water was used, the remarkable difference is that the degradation of phenol gradually leveled off in all the three curves and the transmittance increased with the decreasing flow rate or increasing irradiation time.

Previous report, the transmittance did not change due to the suspension in tap water in the batch cylinder photoreactor was mechanically stirred (4). In this study, at the flow rates lower than $50 \mathrm{ml} \mathrm{min}^{-1}$, the transmittances of the effluents were above $90 \%$, and most of $\mathrm{TiO}_{2}$ particles remained in the tubular photoreactor during the treatments. These results are reasonably explained by the increase in the coagulation which was caused by the electrolytes dissolved in the $\mathrm{pH} 7$ of tap water.

The concentration of $\mathrm{TiO}_{2}\left(\mathrm{C} \mathrm{mg} \mathrm{l}^{-1}\right)$ in the effluent was estimated from the calibration curve obtained between the $\mathrm{TiO}_{2}$ concentration and transmittance (\%) and was as follows: tap water: $T=0.11(C=396) ; T=2.31(C=132) ; T=$ $19.3(\mathrm{C}=47.6) ; \mathrm{T}=72.1(\mathrm{C}=4.22) ; \mathrm{T}=82.4(\mathrm{C}$ $=2.16) ; \mathrm{T}=90.2(\mathrm{C}=1.70) ; \mathrm{T}=95.4(\mathrm{C}=1.39)$; $T=97.3(C=1.27) ; T=98.1 \quad(C=0.75) ;$ deionized water: $T=0.09(C=437) ; T=0.11(C$ $=396) ; T=0.14(C=333) ; T=0.17(C=271)$.

The $\mathrm{pH}$ of the $\mathrm{TiO}_{2}$ suspensions was around 6.5 (isoelectric point (IEP) of $\mathrm{TiO}_{2}$ : 6.4-6.6 (11)). At the IEP, the net interfacial effect shows a maximum attraction resulting in a maximum particle size. Furthermore, common electrolytes $\left(\mathrm{Na}^{+}, \mathrm{K}^{+}, \mathrm{Ca}^{2+}, \mathrm{Mg}^{2+}, \mathrm{Cl}^{-}, \mathrm{SO}_{4}{ }^{2-}, \mathrm{HCO}_{3}{ }^{-}\right)$present in 
the tap water can be bound to the $\mathrm{TiO}_{2}$ particles or very close to its surface, so that they can have significant effects on the interfacial behavior of the $\mathrm{TiO}_{2}$ particles to promote coagulation (11).

Therefore, the removal of phenol did not increase when tap water was used and leveled off with the decreasing flow rate. At a flow rate greater than $50 \mathrm{ml} \mathrm{min}^{-1}$, due to the suspended $\mathrm{TiO}_{2}$ particles were forced into the tubular photoreactor by the hydraulic force of the water flow, the transmittance decreased from $90 \%$ and finally reached below $3 \%$ at the flow rate greater

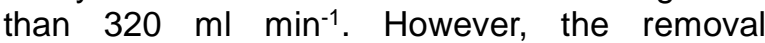
efficiency decreased due to the short irradiation time. To increase it at a high flow rate, it is necessary to decompose the phenol by the circulation mode.

Figure 3 shows the effects of the flow rate on the degradation of phenol $\left(20 \mathrm{mg} \mathrm{l}^{-1}, 795\right.$ $\mathrm{ml}$ ) by $0.75 \mathrm{~h}$-circulative photocatalysis under blacklight irradiation and the transmittance of the effluent flowing from the photoreactor. The irradiation time was always 28.9 min (45 min $510 \mathrm{ml} / 795 \mathrm{ml}$ ) regardless of the flow rate. Tap water and deionized water were used as the solvent of the phenol and the results were compared. When tap water was used, the removal of phenol increased with the decreasing transmittance of the $\mathrm{TiO}_{2}$ suspension.

The removals of phenol $(99.0 \%$, concentration: $\left.0.2 \mathrm{mg} \mathrm{l}^{-1}\right)$ and TOC $(75.0 \%$, concentration: $6.5 \mathrm{mg} \mathrm{l}^{-1}$ ) were a maximum at the flow rate of $600 \mathrm{ml} \mathrm{min}^{-1}$ and the transmittance of $0.26 \%$. These results indicate that the $\mathrm{TiO}_{2}$ particles were perfectly suspended in the narrow pyrex tube at the fastest flow rate. The high flow rate circulating in the tubular photoreactor supported the suspension of $\mathrm{TiO}_{2}$ particles in tap water, while sedimentation of the $\mathrm{TiO}_{2}$ occurred at the low flow rate. On the other hand, when deionized water was used, the removal of phenol was $99.7 \%$ even at the highest flow rate of 600 $\mathrm{ml} \mathrm{min}^{-1}$ and the transmittance was $0.09 \%$ even at the lowest flow rate of $100 \mathrm{ml} \mathrm{min}^{-1}$.

The transmittance in deionized water relatively did not change with the decreasing flow rate. However, a little decrease in the removal was observed with the decreasing flow rate. The high circulative flow rate using deionized water as the solvent of the water pollutants has been reported ${ }^{(3,6,7)}$, although the effect of flow rates was not investigated. The high flow rate supposedly also supported a mixing in the $\mathrm{TiO}_{2}$ suspension. The optimum photocatalysis was achieved at the circulative flow rate of $600 \mathrm{ml}$ $\mathrm{min}^{-1}$ with higher the flow rates not increasing the efficiency significantly. Under sunlight irradiation, the removals of phenol and TOC were $99.3 \%$ and $75.0 \%$, respectively.

The removal efficiency with sunlight irradiation was a slightly higher than that with blacklight irradiation due to a slightly higher intensity of sunlight than that of black light. The photocatalytic treatments were performed with irradiations of both black light and sunlight by turns. Figure 4 shows the removal of phenol (10 I) by the circulative photocatalytic treatment at the flow rate of $430 \mathrm{ml} \mathrm{min}^{-1}$ (runs 1-6) and $600 \mathrm{ml}$ $\mathrm{min}^{-1}$ (runs 7-12), and the results of 4 repeated treatments (12 runs per treatment; one run: 795 $\mathrm{ml})$.

The $\mathrm{TiO}_{2}$ particles were spontaneously sedimented in the separation tank. After one photocatalytic treatment $(10 \mathrm{I})$, the recovered $\mathrm{TiO}_{2}$ slurry was reused for repeated treatments. The first, second, third, and fifth treatments were performed with black light irradiation and the fourth treatment was performed with sunlight irradiation. There was only a slight decrease in the removal in spite of 4 repeated uses of $\mathrm{TiO}_{2}$. The inhibition effect by the electrolytes in tap water was not observed when the effluent was repeatedly recycled at a very high flow rate. On the other hand, the photocatalyst efficiency remained mostly constant in 5 times uses of $\mathrm{TiO}_{2}$ in ultra pure water ${ }^{(3)}$.

\section{CONCLUSIONS}

The circulative flow treatment was preferred over the single pass treatment for the $\mathrm{TiO}_{2}$ suspension photocatalysis in the tubular photoreactor. The circulative flow rate of $600 \mathrm{ml}$ $\mathrm{min}^{-1}$ was sufficient for the suspension photocatalytic treatment and for the effective photocatalysis. At low flow rate, a fraction of $\mathrm{TiO}_{2}$ was sedimented in tap water, but $\mathrm{TiO}_{2}$ was perfectly suspended in deionized water. The $\mathrm{TiO}_{2}$ slurry sedimented by standing was repeatedly reused without decreasing the degradation efficiency. Circulative photocatalytic treatment can be conducted continuously integrating with $\mathrm{TiO}_{2}$ separation under irradiations of sunlight and black light by turns.

\section{ACKNOWLEDGEMENTS}

The author would like to thank Prof. Kiyoshi Hasegawa, Department of Chemical and Biochemical Engineering, Faculty of Engineering, Toyama University for his supervising in the PhD course. 


\section{REFERENCES}

1. D. Robert, A. Piscopo, O. Heintz, J.V. Weber, Photocatalytic detoxification with $\mathrm{TiO}_{2}$ supported on glass-fibre by using artificial and natural light, Catal. Today 54 (1999) 291-296.

2. D. Gumy, A.G. Rincon, R. Hajdu, C. Pulgarin, Solar photocatalysis for detoxification and disinfection of water: Different type of suspended and fixed $\mathrm{TiO}_{2}$ catalysts study, Solar Energy 80 (2006) 1376-1381.

3. P. Fernandez-lbanez, J. Blanco, S. Malato, F.J. de las Nieves, Application of the colloidal stability of $\mathrm{TiO}_{2}$ particles for recovery and reuse in solar photocatalysis, Water Research 37 (2003) 3180-3188.

4. K. Hasegawa, T. Ito, W. Nakamura, M. Nagai, S Kagaya, Electrolyte-promoted easy separation of suspended $\mathrm{TiO}_{2}$ particles with a solids retaining type centrifuge in combination with photoreactor to degrade dibutyl phthalate, Chemistry Letter 32 (2003) 596-597.

5. T.E. Doll, F.H. Frimmel, Cross-flow microfiltration with periodical bach-washing for photocatalytic degradation of pharmaceutical and diagnostic residuesevaluation of the long-term stability of the photocatalytic activity of $\mathrm{TiO}_{2}$, Water
Research 39 (2005) 847-854.

6. J. Gimenez, D. Curco, M.A. Queral, Photocatalytic treatment of phenol and 2,4dichlorophenol in a solar plant in the way to scaling up, Catal. Today 54 (1999) 229-243.

7. S. Malato, J. Blanco, A. Vidal, D. Alarcon, M.I. Maldonado, J. Caceres, W. Gernjak, Applied studies in solar photocatalytic detoxification: an overview, Solar Energy 75, (2003) 329-336.

8. K. Okamoto, Y. Yamamoto, H. Tanaka, M. Tanaka, A. Itaya, Bull. Heterogeneous photocatalytic decomposition of phenol over $\mathrm{TiO}_{2}$ powder, Bull. Chem. Soc. Jpn. 58 (1985) 2015-2022.

9. D. Vione, C. Minero, V. Maurino, M.E. Charlotti, T. Picatonotto, E. Pelezzetti, Degradation of phenol and benzoic acid in the presence of a $\mathrm{TiO}_{2}$-based heterogeneous photocatalyst, Appl. Catal. B 58 (2005) 7988.

10. D. Suryaman, K. Hasegawa, S. Kagaya, Combined biological and photocatalytic treatment for the mineralization of phenol in water, Chemosphere 65 (2006) 2502-2506.

11. W. Xi, S.-U. Geissen, Separation of titanium dioxide from photocatalytically treated water by cross-flow microfiltration, Water Research 35 (2001) 1256-1262.

\section{FIGURES :}
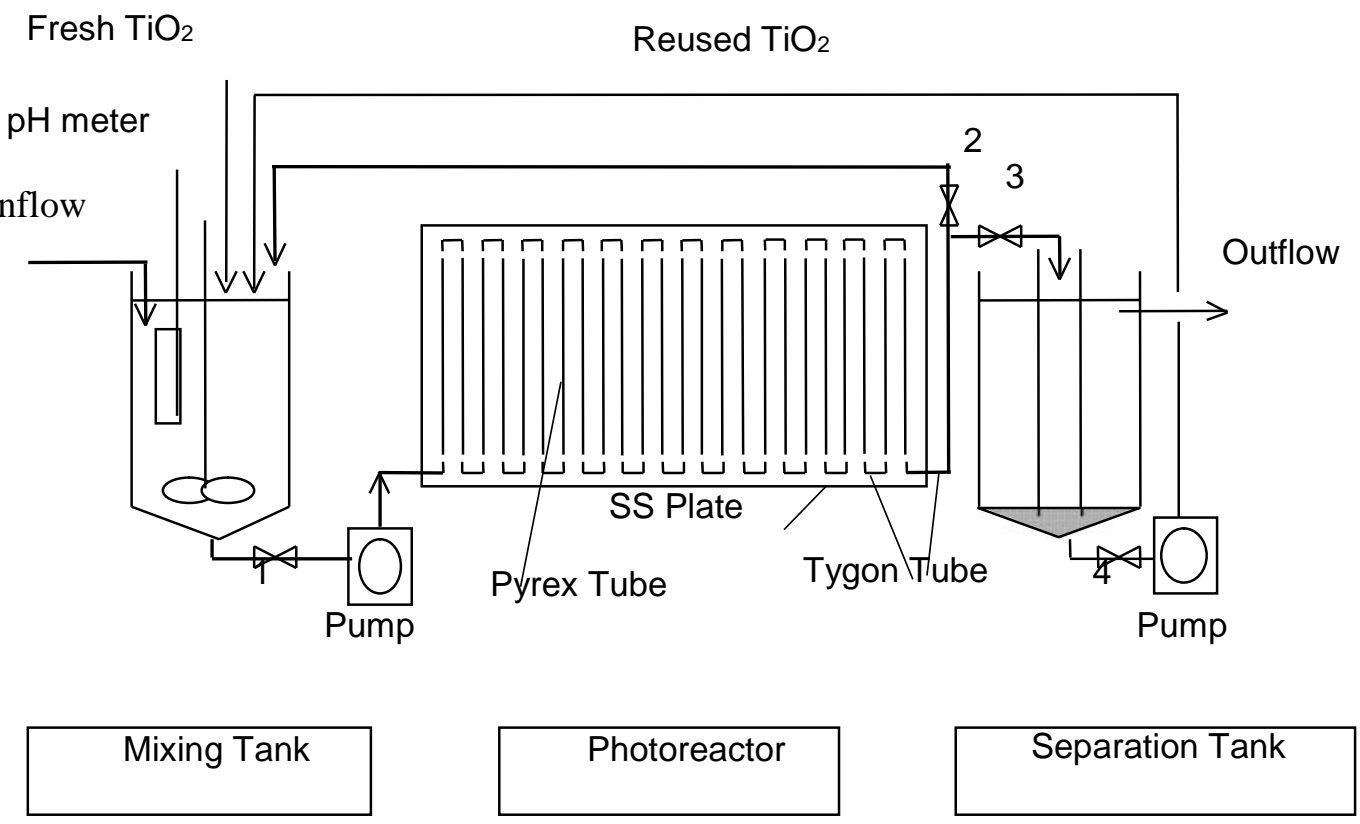

Fig. 1. Schematic of the photocatalytic treatment- $\mathrm{TiO}_{2}$ separation system. 


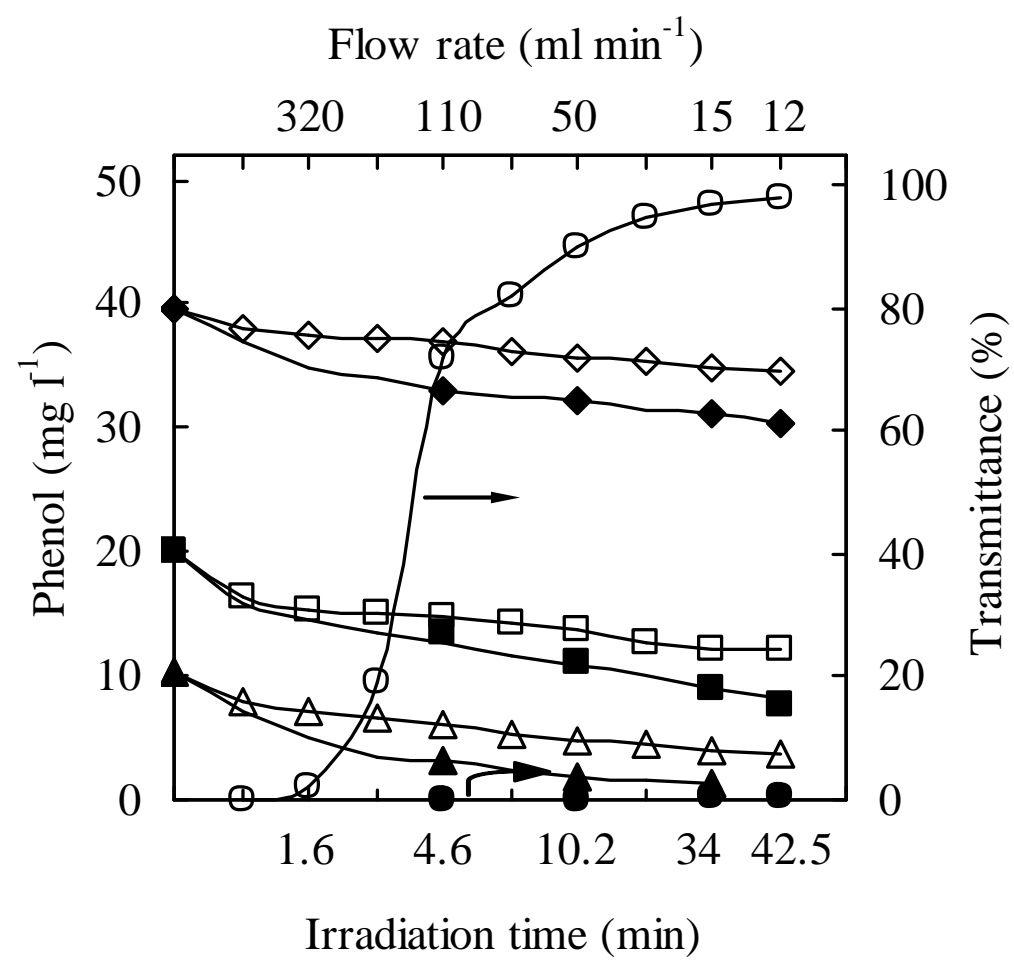

Fig. 2. Time profile of the transmittance and phenol concentration during the photocatalytic degradation of phenol by a single pass flow under black light irradiation. $\bigcirc, \bullet$ : Transmittance; $\downarrow$, $\diamond: 40 \mathrm{mg} \mathrm{l}^{-1} ; \quad, \quad: 20 \mathrm{mg} \mathrm{l}^{-1} ; \quad, \Delta: 10 \mathrm{mg} \mathrm{l}^{-1} ; \diamond, \quad, \Delta, \circ:$ Tap water; $, \quad, \quad, \bullet:$ Deionized water.

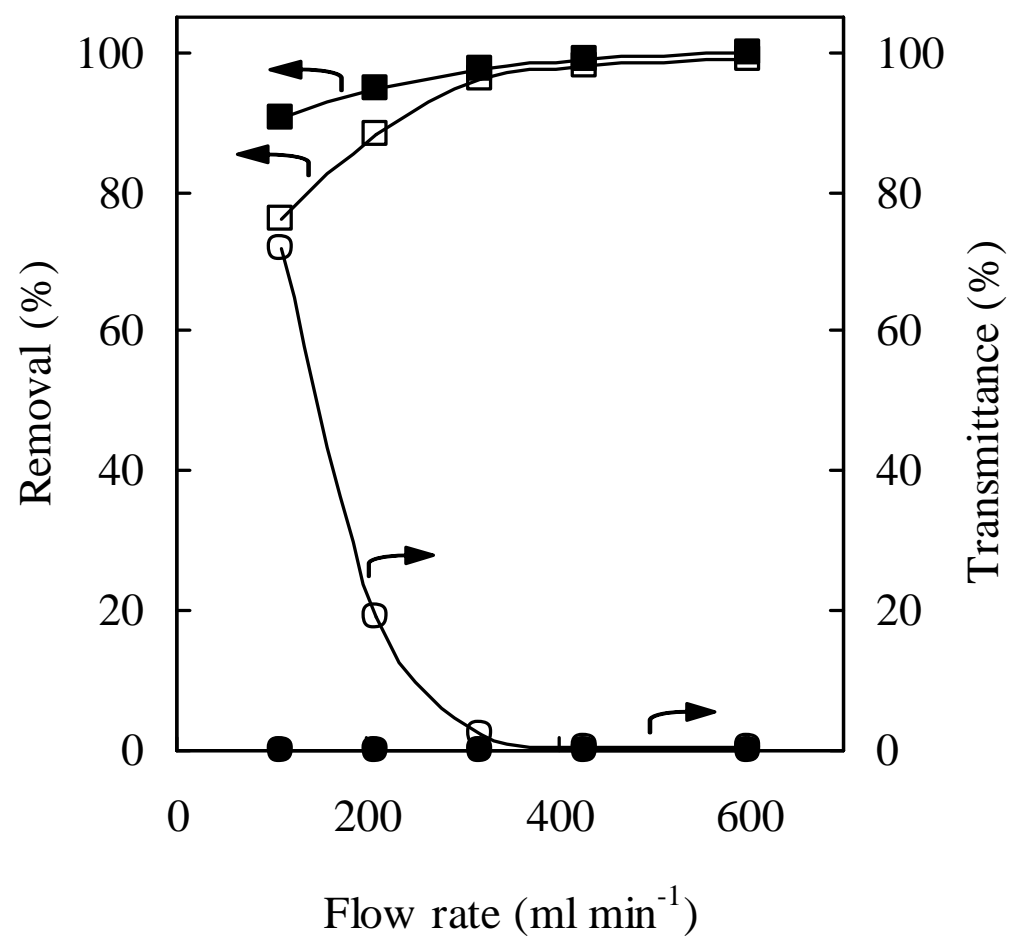

Fig. 3. Removal of phenol for photocatalytic degradation of phenol $\left(20 \mathrm{mg} \mathrm{l}^{-1}, 795 \mathrm{ml}\right)$ under black light irradiation at 45 min circulation flow rates and transmittance of the effluent. $\bigcirc$, : Tap water; $\bullet$ : Deionized water. 


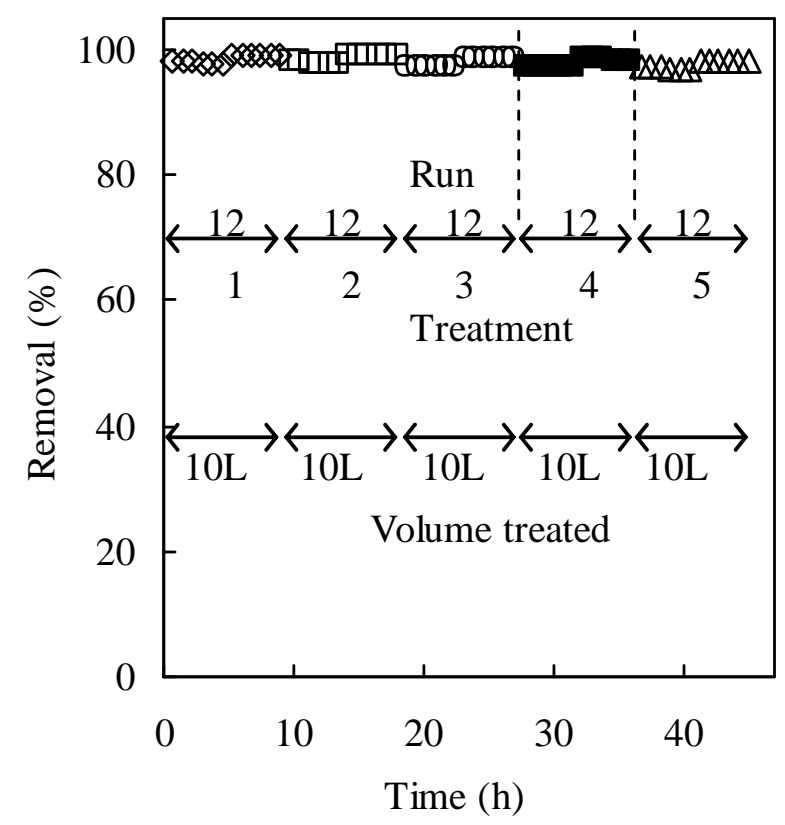

Fig. 4. Photocatalytic degradation of biologically-treated phenol $\left(20 \mathrm{mg} \mathrm{l}^{-1}\right)$ at successive circulation flow rates of 430 and $600 \mathrm{ml} \mathrm{min}^{-1}$ under black light $(\diamond,, \circ, \Delta)$ and sunlight ( ) irradiations. 
Dhanus Suryaman: Titanium Dioxide Suspension Photocatalysis in a Tubular ... JAI Vol.4, No.2, 2008 